COVID 19: Absent bodies in relation to Ernst Neufert's Architect's Data

PALABRAS CLAVE - ARPUITECTURA Y ANTROPOMETRÍA AROUITECTURA Y COVID 19 . EL ARTE DE PROYECTAR EN AROUITECTURA DE NEUFERT . CUERPO Y AROUITECTURA

KEYWORDS - ARCHTECTURE AND ANTHROPOMETRY . SEYWORDS - ARCHITETURE AND ANTHROPOMETRY DATA. BODY AND ARCHTECTURE

\section{RESUMEN}

La pandemia COVID 19 ha instalado condiciones que alteran los modos de habitar el espacio. Una serie de restricciones cuantitativas de distancia física, barreras y limitaciones de aforo en espacios colectivos han proliferado, pasándose a usar al cuerpo humano desde una dimensión meramente antropométrica. Aquí se propone una aproximación por medio de la crítica y las representaciones, confrontando estos hechos con el conjunto de estándares desarrollados por Neufert en su manual ya clásico (Bauentwurfslehre, 1936), quien declaraba allí al hombre como "medida de todas las cosas". Las relaciones de proximidad y ocupación prescritas en dicho manual parecen estar temporalmente suspendidas, y en este ensayo se reconoce ese hecho como una oportunidad visual para, a modo de interpretación, hacer una actualización de sus clásicas representaciones, adaptadas a las nuevas condiciones espaciales, y dar lugar a una reflexión acerca de la suspensión de la vida colectiva y urbana que ello implica, y cómo los cuerpos se han ausentado del espacio público.

\section{ABSTRACT}

The COVID 19 pandemic has installed conditions which alter the ways of inhabiting space. A series of quantitative restrictions on physical distance, barriers, the ways to move and capacity limitations in collective spaces, have proliferated, using the human body merely as anthropometric instrumentalization. Here is proposed an approximation through criticism and representations, confronting these facts with the set of standards developed by Ernst Neufert in his classic handbook (Bauentwurfslehre, 1936), who declared man as "measure of all things". In pandemics, Neufert's relationships of proximity and occupation seem to be temporarily suspended, and this fact is recognized as a visual opportunity to, as interpretation, make an update of its classic representations, adapted to the new spatial conditions, and give rise to a reflection on the suspension of collective and urban life that this implies, and the absents bodies of the public spaces.

\title{
COVID 19: Los cuerpos ausentes a partir del Arte de Proyectar en Arquitectura de Ernst Neufert
}

\author{
JOSÉ IGNACIO VIELMA·Universidad de Chile · jivielma@uchilefau.cl \\ Fecha de recepción: 31 de marzo de 2021 · Fecha de aceptación: 31 de mayo de 2021
}

\section{INTRODUCCIÓN}

La pandemia COVID 19 ha instalado restricciones sanitarias que afectan y modifican la manera en cómo se habita el espacio: distancia física, multiplicación de barreras y limitaciones de aforo en espacios públicos, comerciales y educativos, son algunas de las nuevas condiciones que alteran nuestra relación con el habitar (Shields et al., 2020). Como expresa Gaytán Alcalá (2020), la pandemia Covid es "una crisis sanitaria que ha vaciado las ciudades y llenados las casas", por lo que ha reconfigurado el sentido de la relación entre hogar y mundo. En este contexto se han dado a gran velocidad transformaciones socioespaciales muy diversas, y grandes desafíos, pero tambien oportunidades que incluyen el replanteamiento de estándares de diseño urbano hacia modelos inteligentes, resilientes, inclusivos y atentos a la salud y a las relaciones sociales -y no a su supresión- (Gesto, 2020). La cultura instrumentalista de la disposición y usos del espacio ha proporcionado soluciones basadas en la restricción y nuevas formas de organización del espacio y el tiempo -distanciamiento, aforos, cuarentenas, etc.-. Sin embargo, se empieza también a pensar en necesidades de rehabitar de modos distintos. Por ejemplo, entornos tan instrumentalizados como los hospitales que se han visto sometidos a grandes exigencias, en simultáneo son objeto de reconsideraciones hacia modos más humanizados de ser habitados (Pernas
Galí, 2020). Pero persiste que los espacios clásicamente colectivos parecen estar destinados al despoblamiento y el control, con poca reflexión acerca de sus modos de habitar. En los espacios públicos y colectivos, más que sujetos, han proliferado las marcas y señales de dónde los cuerpos pueden ubicarse, dándose una paradóiica experiencia de cuerpos ausentes que solo existen por la presencia de la marca de su ocupación prescrita.

En 1936, en el Arte de Proyectar en Arquitectura (Baventwurfslehre) de Ernst Neufert, el arquitecto alemán desarrolla un sistema de estándares para el diseño arquitectónico, declarando que comprende al cuerpo humano como "medida de todas las cosas". A lo largo del volumen se elaboran esquemas e ilustraciones donde las dimensiones y la forma de ocupar los espacios proviene de una comprensión antropométrica del cuerpo, de sus partes y de los objetos cotidianos que lo rodean. Allí la representación de la figura humana es esquemática o reducida a lo dimensional, sin dar cuenta de su relación con el espacio arquitectónico entendido como fenómeno. En el contexto de la pandemia, muchas de las prescripciones dimensionales del Neufert parecen estar suspendidas temporalmente, y en ese contexto este trabajo explora si las nuevas normas consecuentes de la contingencia no son sino una exacerbación de un modo de comprensión que ya venía manifestándose en declive. Por lo tanto, estas normas y restricciones no logran dar cuenta 
de las complejas variables que se juegan en el habitar del espacio arquitectónico y urbano. Ante la proliferación de esquemas y aplicaciones gráficas en los medios de comunicación o directamente en el espacio público para recordar al habitante como actuar y disponer su cuerpo respecto a la norma, se identifica un reflejo de la manera como el manual de Neufert persiste como forma de pensar y representar la relación entre cuerpo y espacio. En este artículo se reconoce ese hecho como una oportunidad visual para, a modo de interpretación -y no de prescripción-, se puedan actualizar las clásicas representaciones del Neufert, adaptadas a las nuevas condiciones de la ocupación y el uso del espacio, y dar lugar a una reflexión acerca de la suspensión de la vida colectiva y urbana que ello implica.

\section{METODOLOGÍA}

Se propone una revisión desde la crítica arquitectónica de dos aspectos vinculados por la pandemia. Primero, cómo ciertos discursos de la arquitectura moderna han comprendido la relación entre cuerpo y arquitectura de una manera limitada a lo antropométrico, el cuerpo humano únicamente como unidad dimensional. Segundo, la manera cómo en el contexto de la pandemia, la necesidad de controlar los contactos entre las personas ha llevado a una serie de prescripciones acerca del comportamiento del cuerpo en el espacio, y mayormente, de la necesidad de distancia o de barreras entre ellos. Para esto, reconociendo al Arte de Proyectar en Arquitectura, de Ernst Neufert como ejemplo de la normalización de las relaciones entre cuerpo y arquitectura, se propone una revisión crítica de éste por medio de una serie de representaciones vinculadas a él. El texto crítico se desarrollará a partir de referencias clásicas y contemporáneas acerca de esta relación, y a la exploración de las relaciones del manual alemán con su momento histórico y las valoraciones implícitas. Adicionalmente, se contrastarán sus representaciones originales respecto a las realizados para esta investigación, proponiendo lado a lado una imagen fascimilar del Neufert pre-COVID 19, conocido por todos, y una nueva imagen que reinterpreta al Neufert y especula sobre una edición actualizada a la pandemia. Para mantener la consistencia entre las propuestas y los originales, las claves típicas de representación del manual alemán -las maneras de dibujar el espacio y los cuerpos - los tratamientos de las dimensiones y la tipografía- son replicadas. Por medio de lo anterior, se lograría consolidar un discurso crítico que une texto y representaciones, y que toma como evidencia las relaciones del contexto histórico del libro de Neufert, las representación cada vez más frecuente en los medios de comunicación de los cuerpos en el espacio, y las teoría sobre las relaciones entre cuerpo y arquitectura.

\section{DISCUSIÓN DE ANTECEDENTES}

\section{CUERPOS Y AEROSOLES}

La representación de las relaciones entre el cuerpo y el espacio ha sido frecuente para comentar y prescribir los modos de ocupación en el contexto de la pandemia COVID 19. El 28 de octubre de 2020 el diario El País de España publicó en su edición digital un reportaje con una infografía titulada "Un salón, un bar y una sala de clase: así se contagia el coronavirus en el aire" (Zafra \& Salas, 2020b). En una semana el contenido fue visitado desde 12 millones de dispositivos y se posicionó como el más visto en el período. Había sido antecedido por otro reportaje del 8 de junio de 2020, "Radiografía de tres brotes: así se contagiaron y así podemos evitarlo" (Zafra \& Salas, 2020a), que describía la relación entre espacio, ocupación y circulación del aire con los contagios producidos en una oficina abierta, un restaurante y un autobús. Ambas notas, a partir de investigaciones científicas interdisciplinarias de Estados Unidos, China y Corea, reconocen el rol de los aerosoles formados por las partículas provenientes de la respiración en los contagios, por su capacidad de quedarse en suspensión y de moverse con leves corrientes de aire. Los periodistas refirieron que, al revisar el resultado y las descripciones de las investigaciones, reconocieron una "oportunidad visual" para explicar un tema sanitario para todo público. El modo en que esto fue explicado gráficamente no es muy distinto a los modos en que la arquitectura moderna ha representado las relaciones entre cuerpo y espacio

La nota de octubre abre su explicación gráfica con una secuencia de cuatro cortes, en perspectiva, de una figura humana femenina que ocupa un espacio cúbico. De ella emanan vapores de color naranja y, a medida que se indica el paso del tiempo -5 , 20,40 y 60 minutos-, esa nube que expande el virus, se hace más densa y extensa. Se puede reconocer una relación formal entre estas representaciones y el dibujo clásico de Leonardo Da Vinci del cuerpo basado en las prescripciones de Vitrubio. El cuerpo ideal del Renacimiento quedaría sustituido en esta secuencia por un cuerpo contemporáneo, contaminante (FIGURA 1). Sus dimensiones internas y sus relaciones de proporción no son ni importantes, ni ideales. Su alcance ya no es el del círculo o el cuadrado que definen el límite de sus extremidades. El espacio que ocupa su influjo es inestable y variable, depende de la intensidad con la que hable o de su ritmo respiratorio, del tiempo que permanezca en un espacio o de imperceptibles corrientes de aire a su alrededor.

En el resto de las representaciones de ambas notas utilizan la perspectiva isométrica -representación proyectiva típicamente arquitectónica- para explicar las relaciones entre los cuerpos y el espacio. En las isométricas de El País, los espacios son los cotidianos: la sala de clases, el restaurante, el transporte público, la oficina abierta, el cuarto de estar o el bar. Son representaciones espaciales que ilustran el proceso de contagio, habitadas por cuerpos que son idénticos entre sí, pero que son clasificados en sanos y enfermos, con mascarilla y sin mascarilla, pacientes cero o pacientes sucesivos. A medida 
1. Izquierda: El Hombre de Vitrubio, Leonardo Da Vinci, 1492. Derecha: Esquema de grados de concentración de aerosoles provenientes de la respiración por la presencia de un cuerpo en un espacio cerrado. Fuente: El País (España), 28 de octubre de 2020.

2. Dibujos isométricos indicativos de la presencia de cuerpos humanos en espacios de uso cotidiano (salón y sala de clases) y los procesos o riesgos de contagio. Fuente: El País (España) 6 de junio y 28 de octubre de 2020.

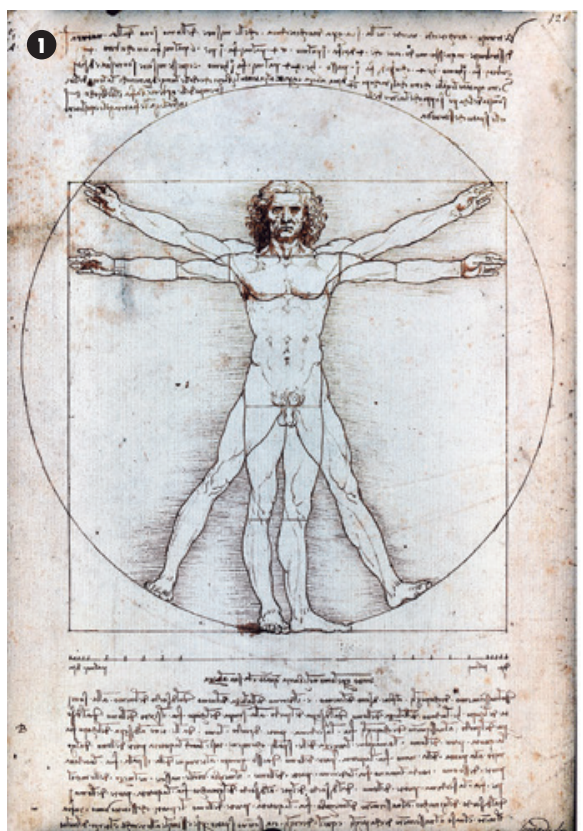

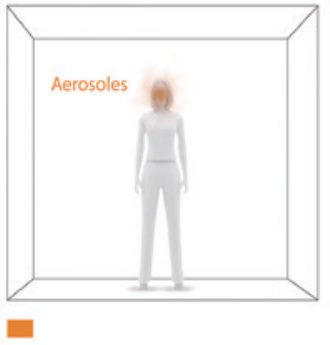

5 minutos

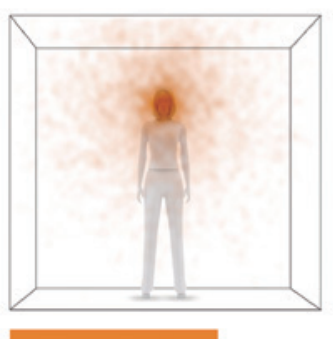

40 minutos

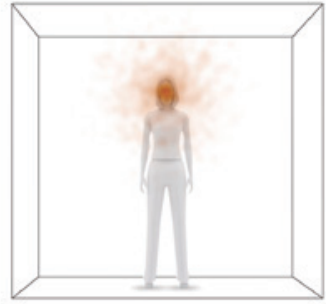

20 minutos

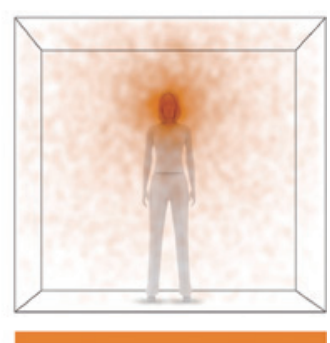

60 minutos

\section{(2) $12.00 a 16.00$}

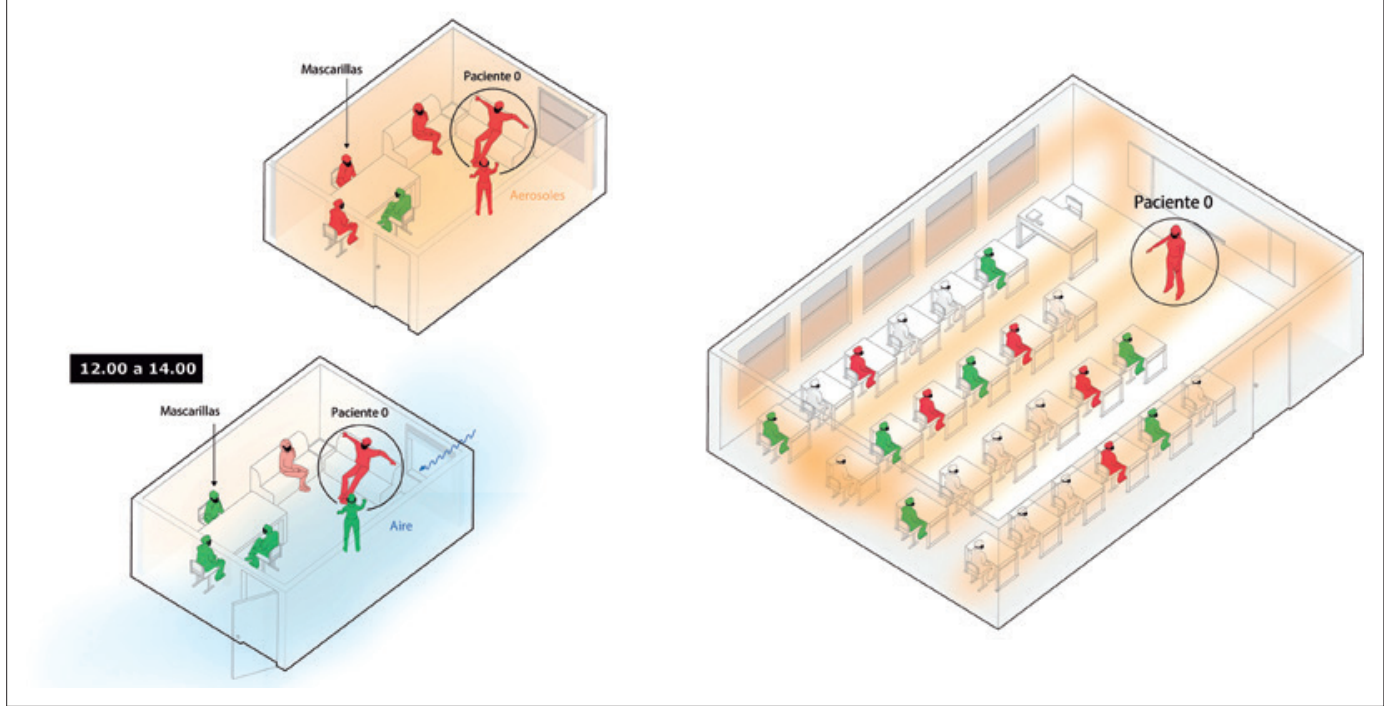

que se contagian, los cuerpos cambian de color: rojo para los enfermos, grises para los aún no expuestos, verdes para los expuestos y no contagiados. A las ilustraciones se suman elementos adicionales como las ventanas y equipos de climatización, y su capacidad de colaborar en el recambio de aire, así como señales que indican los flujos de aire, distancias y dimensiones (FIgura 2).

\section{CUERPO Y ARQUITECTURA}

Como desarrolla Ignasi de Solà-Morales (1996), el cuerpo ideal y canónico, mayormente masculino, fue modelo para la arquitectura clásica, la cual remitía a sus proporciones, a su descomposición analítica y a veces a su propia forma. Según Zöllner (2014), el cuerpo funcionaba a la vez como metáfora y como modelo morfológico: "ambos, el cuerpo y el edificio estaban definidos y transcritos metafóricamente con la ayuda de medidas, números, proporciones y figuras geométricas" (pp. 48-49). Con el desarrollo del pensamiento moderno y las arquitecturas consecuentes, se estableció una dialéctica entre un cuerpo que no debía salirse de la norma, que no podía en ningún caso volverse Otro, y una arquitectura que se especializaba en corregir, educar, consensuar, reprimir o castigar al cuerpo que exceptuaba lo saludable o lo normativo. Es el momento de la emergencia de las instituciones modernas de educación, adiestramiento militar, saneamiento y tratamiento de la locura. Esto derivó hacia los modelos higienistas y funcionalistas de una arquitectura que se esmeró en adecuarse a los distintos órganos y funciones. Un cuerpo descompuesto analítica y mecánicamente busca, en la arquitectura y en la ergonomía -la adaptación para los órganos-, respuestas para la higiene, la fatiga, la posición óptima o el espacio mínimo necesario. La arquitectura se concibe, según continúa Solà-Morales, como una enorme prótesis que resuelve la necesidad de cuerpos que se fragmentan y dispersan, a la vez que se hacen productivos y estandarizados, produciendo, paradójicamente, una suerte de "cuerpos ausentes".

\section{HIGIENISMO Y ARQUITECTURA MODERNA}

Reuniendo estas observaciones con las infografías referidas al inicio, se identifica una reaparición en el campo de las representaciones de prensa y al alcance del gran público, de la relación entre cuerpo y espacio arquitectónico. Como es de esperarse, en el contexto de 
la pandemia COVID 19 se recupera un discurso higienista que buscaba legitimar de modo determinista a la arquitectura (Palero \& Avila, 2020). Esta relación determinista entre pandemia y arquitectura tiene al parecer antecedentes directos. Se refiere que Le Corbusier pasó la mayoría del tiempo recluido en su departamento en París durante la gripe española en 1918, atendiendo consejos médicos de la época sobre beber cognac y fumar puros (Ortiz de Lejarazu, 2018). En el período inmediato a esa fecha publicó por medio de L'Espirit Nouveau gran parte de la doctrina que determinó sus primeras obras arquitectónicas puristas, que radicalizaban su práctica proyectual. Allí, la razón higienista fue muy útil como justificación a la limpieza de la forma, los grandes vanos para el sol y el aire o las distancias entre los edificios, pero también para justificar la distancia entre las personas. Los edificios modernos debían promover la buena salud y la adecuada moralidad. Un ejemplo es el conocido dibujo en perspectiva de las Immeuble-Villas (1922-28), donde un saludable y activo sujeto golpea una pera de boxeo colgada mediante una cuerda tensa que recorre la doble altura de una gran terraza, con vistas a prados y colinas, mientras que, desde el segundo piso, una mujer parece hacer una pausa en el aseo de una alfombra colgada en la barandilla, para observarlo amorosamente. En este dibujo quedarían retratadas las relaciones entre cuerpo, espacio, arquitectura y salud propios del movimiento moderno. El espacio se presenta amplio, aireado y luminoso. Sus habitantes hacen deporte, usan los espacios exteriores, se hace el aseo con facilidad. De modo similar, la Ville Savoye todavía hoy recibe a los visitantes con una alfombrilla limpiapiés integrada al pavimento frente a la puerta de ingreso, y un lavamanos al lado de la rampa que da acceso al segundo piso está disponible para ser usado en todo momento. Ambos dispositivos, bien integrados a la vivienda, serían muy apreciados en el contexto actual.

El higienismo instauró una comprensión del cuerpo como agente contagioso o contagiable, dando lugar a un modo de proyectar instrumentalizado, mientras los cuerpos arquitectónicos han mutado en artefactos incorpóreos, fragmentados, anónimos o desproporcionados. Persiste la necesidad de una arquitectura que pueda ser capaz de reconocer un cuerpo precario e insistentemente humano, fragmentado, pero dinámico y cambiante. En un sentido similar, Rob Imrie (2003) critica que el cuerpo haya sido frecuentemente usado por la arquitectura solo como estándar o referencia dimensional. Los cuerpos son sujetos: "sensibles-sintientes, comunicativos, prácticos e inteligentes", y no solo objetos dispuestos a obedecer las leyes de la física. En consecuencia, La arquitectura, desde la fenomenología, debería reconocer a cada cuerpo como un modo de estar en el mundo. Esto es un modo radicalmente distinto de proyectar que cuando se le piensa en tanto que cuerpo que sólo ocupa un espacio.

\section{LOS CUERPOS OCUPANTES DE ERNST NEUFERT}

El desarrollo de la arquitectura moderna tuvo sus propias versiones de las teorías antropomórficas. Según Zoellner (2014), la industrialización de la construcción, derivada de las aplicaciones tecnológicas y tipológicas, que simplificaban la arquitectura al extremo, estimularon la búsqueda de un cuerpo universal como sujeto para lo edificado. Un ejemplo conocido fue el Modulor de Le Corbusier (1948), propuesto como ejemplo para la producción de edificios y partes asociadas dimensionalmente a un canon. Pero, previamente, un arquitecto alemán había insistido con una serie de modelos dimensionales relacionados a un cuerpo humano estandarizado y serial.

Ernst Neufert, alumno de Walter Gropius en la Bauhaus, es mayormente conocido por ser el autor de Arte de Proyectar en Arquitectura (Neufert, 2013), publicado originalmente en alemán en 1936 como Bauentwurfslehre. Este es un manual de estándares dimensionales y constructivos para el diseño arquitectónico que ha servido de base y de reflejo de la exhaustiva normalización alemana de la arquitectura y la edificación, publicándose en 18 idiomas, y con una circulación actual de 800.000 ejemplares. En Alemania ha alcanzado la $42^{a}$ edición (2018), y en español la 16 ${ }^{a}$ (2013). El Neufert, como se le llama coloquialmente en español, sigue manteniendo casi sin transformación mucho de su contenido original. En él se pueden encontrar desde las recomendaciones de organización de edificaciones complejas como escuelas, fábricas u hospitales, a partir de ejemplos europeos, pasando por las dimensiones ideales de espacios y recintos, hasta las medidas en los tres ejes espaciales de un cerdo o una gallina. Para actualizarse el libro ha añadido importantes contenidos de accesibilidad universal, arquitectura bioclimática, sostenibilidad ambiental y energética y aspectos conceptuales del dibujo asistido por computador.

En una sección que ha estado siempre al inicio en las distintas ediciones, subtitulada El hombre como unidad de medida - La medida de todas las cosas, el autor expone un conjunto de relaciones dimensionales entre el cuerpo, solo masculino, y el espacio arquitectónico, destacando al cuerpo humano como la "medida" correcta. Aquí plantea una de sus tesis más importantes en este trabajo: una estricta prescripción de las dimensiones de la arquitectura en relación con la dimensión del cuerpo, específicamente "el de una persona bien formada y qué espacio ocupa en diferentes posiciones y al moverse" (p. 38). Neufert presentará en las páginas subsiguientes un estudio dimensional del cuerpo en diferentes posiciones, y de cómo cabe en variados espacios según su actividad. A lo largo de todo el libro continuará con gran cantidad de ilustraciones sobre las recomendaciones dimensionales o de organización de los espacios que contendrán a los cuerpos o al mobiliario o accesorios que remiten directamente al cuerpo ocupante del espacio.

La convicción de Neufert con lo dimensional estaba tan arraigada que desarrolló un modelo antropométrico propio, y fue impulsor del uso 
3. Relaciones antropométricas propuestas por Ernst Neufert basada en su teoría de coordinación modular con el módulo octométrico. Publicado originalmente por Ersnt Neufert en Bauordnungslehre (1943). Fuente: Zöellner, 2014.
3

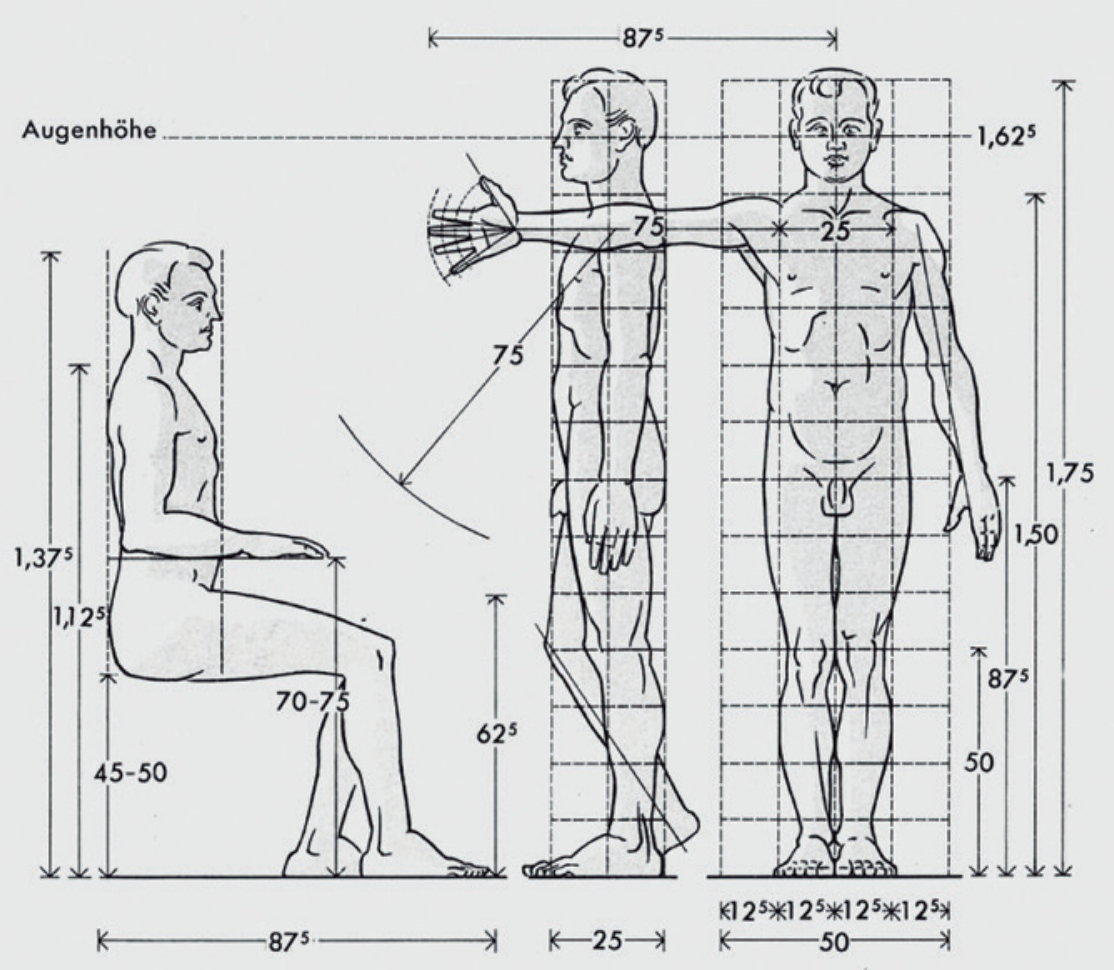

de modelos provenientes de la dimensionalidad del cuerpo en la Alemania Nazi, asociando la construcción con un modelo de cuerpo idelizado (Vossoughian, 2020). Este modelo partía de un cuerpo masculino subdividido en su altura en catorce partes y en su ancho en cuatro. Todas las partes medían $125 \mathrm{~mm}$, y a cada línea de la grilla resultante le correspondía un hecho notable del cuerpo: por ejemplo, la altura de los ojos, los hombros, los genitales o las rodillas (Figura 3). Esta estandarización del cuerpo en una grilla regular, publicado en 1943 en su otra influyente obra, el Bavordnungslehre, acompañaba la máxima obsesión dimensional de Neufert: el desarrollo del Octómetro (1943), un sistema universal de coordinación modular para los materiales constructivos y todo lo edificado, capaz de abarcar como módulo de control, desde el ladrillo hasta las fabricas de armamento o los hangares de la Luftwaffe, y que terminó imponiéndose como estándar general en la Alemania de la época (Imrie, 2003; Vossoughian, 2015, 2016).

Estos desarrollos, y esta ideología, coincidieron con la carrera de Neufert dentro del régimen nacionalsocialista alemán. Allí fue jefe de un departamento con su propio nombre (Abteilung Neufert) en la Inspección General de Edificios de Berlín, que estaba a cargo de Albert Speer,' entre 1938 y 1941. Después fue consultor permanente sobre estándares

\footnotetext{
Albert Speer prologó el Bavordnungslehre de Erns Neufert con las siguiente palabras: "la guerra total demanda la concentración de todas nuestras fuerzas, incluso en la industria de la construcción. La estandarización extensiva para economizar recursos técnicos y para el desarrollo de una producción serial racionalizada es una precondición para un aumento de lo creado, lo cual es necesario para el cumplimiento de nuestros grandes objetivos en la edificación" (citado en Zoellner, traducción propia).
}

arquitectónicos y de construcción (Vossoughian, 2016) desde donde defendió consistentemente la necesidad de la estandarización en pro de la "guerra total" y para lograr los "objetivos en el Este" en la conquista del Lebensraum. Como concluye Zöllner, esta estandarización del cuerpo a partir de un módulo reticular, y no de un sistema de proporciones adaptable a múltiples dimensiones, refleja la idealización de un hombre modélico acorde régimen político en que se desarrollaban sus labores (Zöllner, 2014). Para Neufert, en el contexto del régimen nacionalsocialista en plena guerra, la estandarización dimensional del cuerpo, del edificio o de la construcción, era un modo de pensar necesario para el dominio del espacio. Pero a la inversa, y también de modo útil a la ideología imperante, a partir de sus propias teorías y muy especialmente del modo de representar gráficamente los órdenes prescritos, el espacio era también un instrumento para el dominio de un cuerpo limitado, asociado a una dimensión estándar y a unos modos específicos de ubicarse y posicionarse.

\section{RESULTADOS DE LAS REPRESENTACIONES}

En este apartado, como operación de investigación, se realizará una serie de comparaciones entre reproducciones de las representaciones originales del manual de Neufert y nuevas representaciones realizadas por medio de sus modos expresivos particulares, adaptándose al contexto de la pandemia. Para insistir en la analogía propuesta, se ha utilizado un tipo de dibujo que reproduce el carácter de la representación original, utilizando el mismo tipo de trazo, patrones, tipo de figuras humanas, tipografía y uso de las dimensiones que las utilizadas por décadas en el manual. Cada ilustración nueva corresponde a una ilustración original ubicada en una página específica, seleccionándose una serie de casos representativos de la relación cuerpo-espacio. Las recomendaciones respecto a la pandemia COVID-19 aplicadas a los dibujos actuales provienen de las resoluciones de los organismos 
chilenos y de lo recogido en terreno (Ministerio de Educación-Chile, n.d.; Ministerio de SaludChile, n.d., 2020)

La Figura 4 recoge algunas consideraciones iniciales de Neufert sobre la cabida de los cuerpos en el espacio. Si la representación original en planta elimina completamente la distancia entre los sujetos que ocupan al máximo el espacio disponible, la reinterpretación en versión pandemia que se hace de ella se nota incómodamente vacía, con cuerpos que habitan en solitario o a distancias socialmente inertes. En los alzados interpretativos, los cuerpos solitarios o evitando la interacción, dan cuenta con más fuerza aun de la preeminencia del vacío que anula la relación.

La aparición de una serie de rituales de desinfección relacionados con el cómo llegar a casa, a veces establecidos sin base científica, se refleja en la Figura 5. La figura original de Neufert, en ausencia de COVID-19, muestra el espacio del recibidor de una vivienda desde una lógica moderna. Una persona recibe en su casa a una pareja que llega de visita. La anfitriona ayuda a su invitado a quitarse el abrigo, el cual quedará convenientemente guardado en el guardarropa. Este recibidor es estanco con respecto al resto de la casa, está separado por una puerta que, en este caso, permanece abierta. La reinterpretación para el escenario COVID-19 que se representa busca responder a distintas acciones que las personas han tomado, basada en testimonios sobre los rituales de llegada a la casa en medio de la pandemia causada por el virus. El sujeto llega solo, limpia sus manos, tiene un baño de acceso directo con lavabo y ducha, donde puede desinfectar por completo su cuerpo y dejar su ropa sucia. Además, se cuenta con un espacio para dejar las compras y los objetos que vienen del exterior, mientras que la puerta que conecta al resto de la vivienda permanece cerrada. Es una representación extrema de lo específicos que tendrían que ser los cambios en la vivienda y los modos de vida por la extensión de la situación actual, si se confirmara la necesidad de los modos de vida adoptados por muchos.

4. Organizaciones del cuerpo en espacios mínimos. Arriba: Basado en Neufert (2013). p. 41. Abajo: Interpretación gráfica de las recomendaciones y dimensiones según el contexto de la Pandemia COVID 19. Fuente: dibujos del autor.

5. Izquierda: Recomendaciones para espacio de acceso a la vivienda. Basado en Neufert (2013). p. 159. Derecha: Interpretación gráfica de las recomendaciones y dimensiones según el contexto de la Pandemia COVID 19. Fuente: dibujos del autor.

(4)
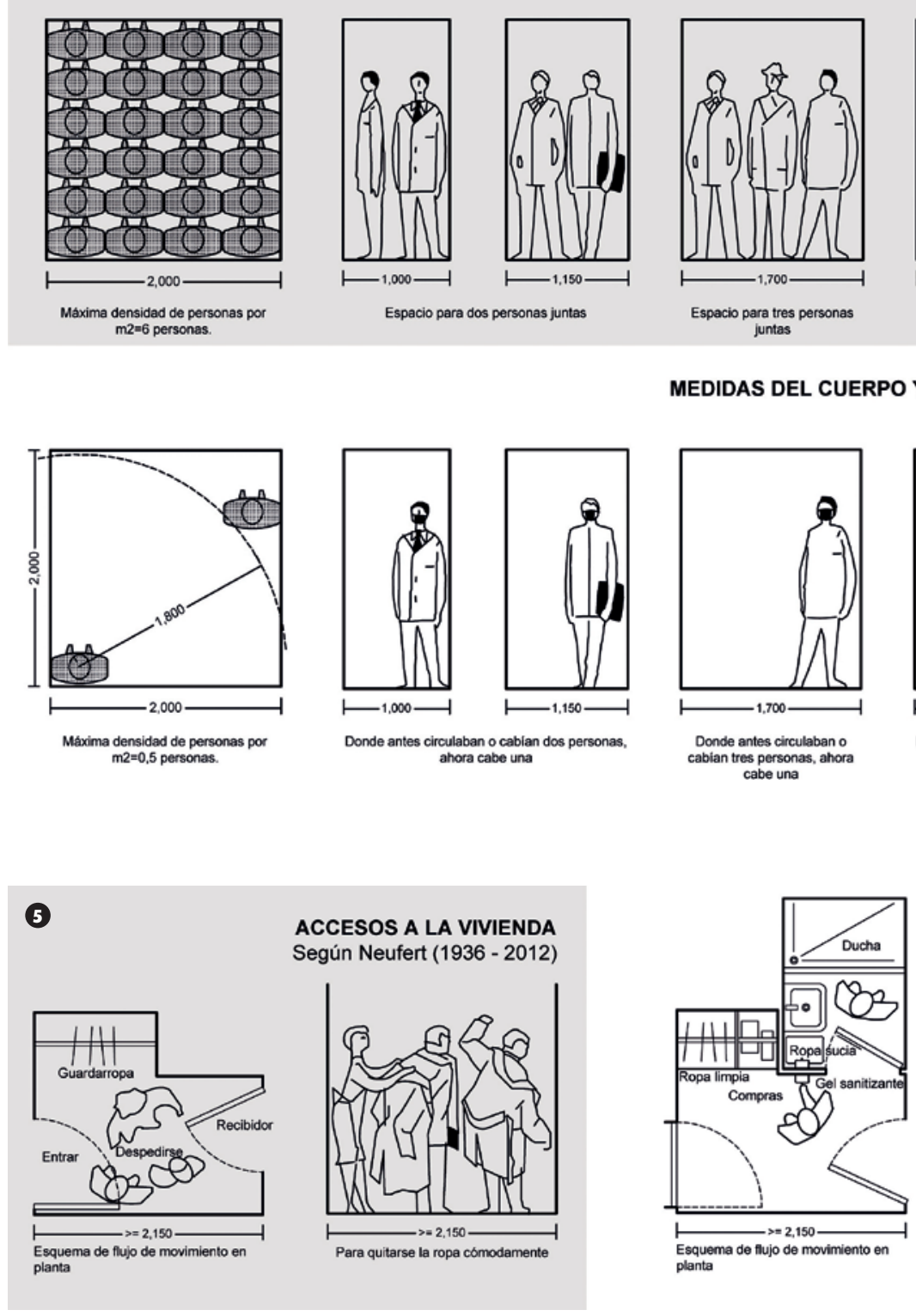

ACCESOS A LA VIVIENDA Variación COVID 2019

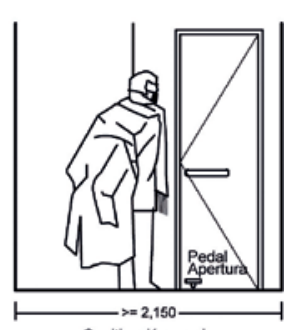

Sanitización previa
MEDIDAS DEL CUERPO Y ESPACIO NECESARIO Según Neufert (1936 - 2012)

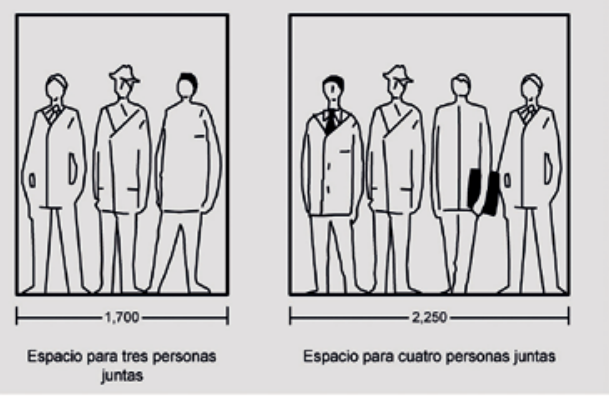

MEDIDAS DEL CUERPO Y ESPACIO NECESARIO
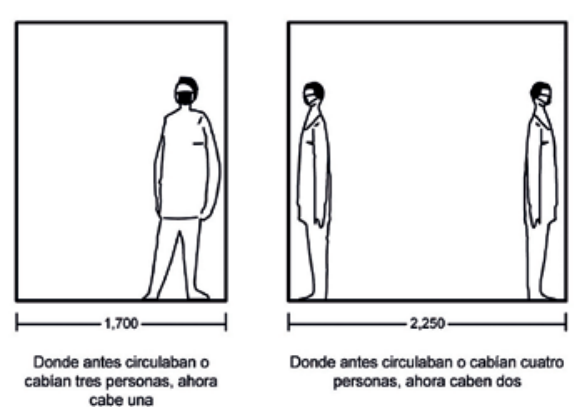

En otro sentido, la Figura 6 sintetiza el cambio radical que se da en la relación entre el comercio y el espacio colectivo que le antecede. En la figura de Neufert, una puerta siempre abierta vincula el espacio exterior con el interior, indicando una accesibilidad inmediata. Sin embargo, en la alternativa durante la pandemia, aparece el espacio de la fila de espera, ahora cotidiano para no exceder el aforo de los espacios comerciales. La demarcación de la distancia entre los cuerpos en el suelo, la desinfección previa de las manos, e incluso, la instalación de dispositivos técnicos que automaticen la apertura de las puertas, evitando ser tocadas, son también nuevas condiciones solicitadas. 
Para recintos cerrados como las salas de clase, se recomienda, aparte del control de aforo que implica la reducción radical del tamaño del grupo, un intercambio de aire a una tasa de seis veces por hora (Harvard Healthy Building Program \& Health, 2020), lo que ha implicado un desafío mayor para los espacios con fines educacionales. En la representación prescriptiva de Neufert (FIgura 7) se expresa una alta cantidad de alumnos y una corta distancia entre ellos. Así mismo, un lavabo para el eventual lavado de manos y un armario común para dejar mochilas o útiles. La alternativa representada en base a las exigencias actuales requiere una distancia entre alumnos muy superior a la convencional. Adicionalmente, el gráfico señala condiciones ideales en las que se pueden agregar lavabos, geles desinfectantes, ventilación mecánica, separar flujos de entrada y salida y la eliminación de armarios comunes para ampliar el tamaño de la sala y reducir la interacción entre los cuerpos. El resultado previsible es una gran disminución de la sinergia entre los alumnos y de la posibilidad de una masa crítica para el curso.

La FIGURA 8 revisa el aforo en los locales de servicio de comida. Neufert proponía un apretado comedor para casi 50 personas. Hoy en día, para adaptarse a las nuevas capacidades recomendadas (entre $25 \%$ y $50 \%$ del aforo original, según las distintas resoluciones del MINSAL en 2020) sirve a no más de 16 personas. Adicionalmente, se incluye la recomendación del uso de ventilación mecánica para garantizar cambio de aire. Sumando a estas variables, la recomendación explícita tiempos máximos en el comedor de dos horas por comensal. Así, el vacío se hace evidente, dando cuenta de un local despoblado, con mesas enormemente distanciadas

El comercio callejero de ferias y similares, consideraba en las ilustraciones prescriptivas de Neufert, el privilegio de la cercanía del vehículo. Se asumía un espacio mínimo para la circulación de personas y una relación abierta entre clientes y feriantes. En la FIGURA 9 se compara lo prescrito por Neufert con una

6. Izquierda: Recomendaciones para acceso sencillo a tienda. Basado en Neufert (2013). p. 268. Centro y derecha: Interpretación gráfica de las recomendaciones y dimensiones según el contexto de la Pandemia COVID 19. Fuente: dibujos del autor.

7. Izquierda: Recomendaciones de ocupación de aulas generales en escuelas básica. Basado en Neufert (2013). p. 203. Derecha: Interpretación gráfica de las recomendaciones y dimensiones según el contexto de la Pandemia COVID 19. Fuente: dibujos del autor.

8. Izquierda: Recomendaciones de ocupación de comedores en negocios de servicio de comida a la mesa. Basado en Neufert (2013). p. 187. Derecha: Interpretación gráfica de las recomendaciones y dimensiones según el contexto de la Pandemia COVID 19. Fuente: dibujos del autor.

(

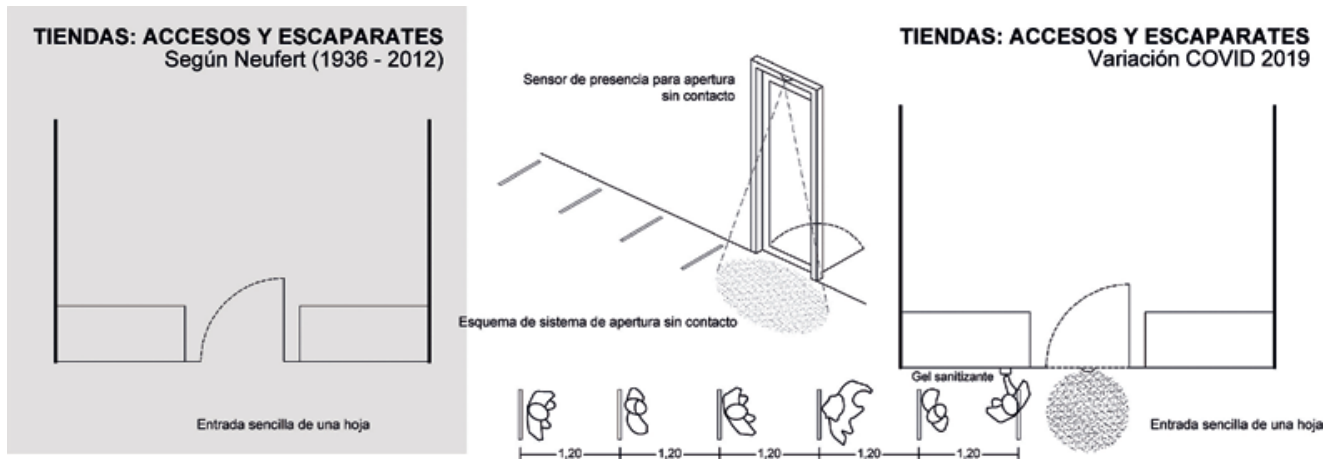

(
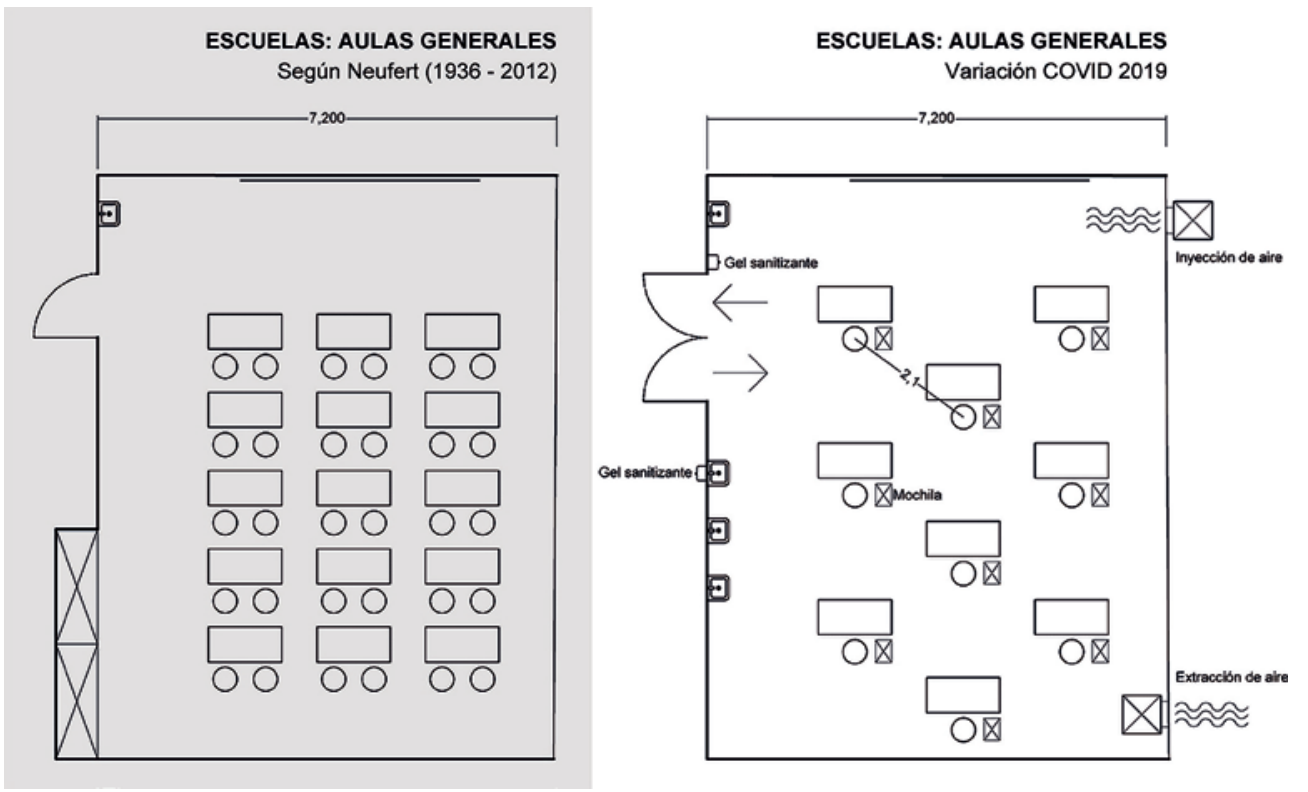

8

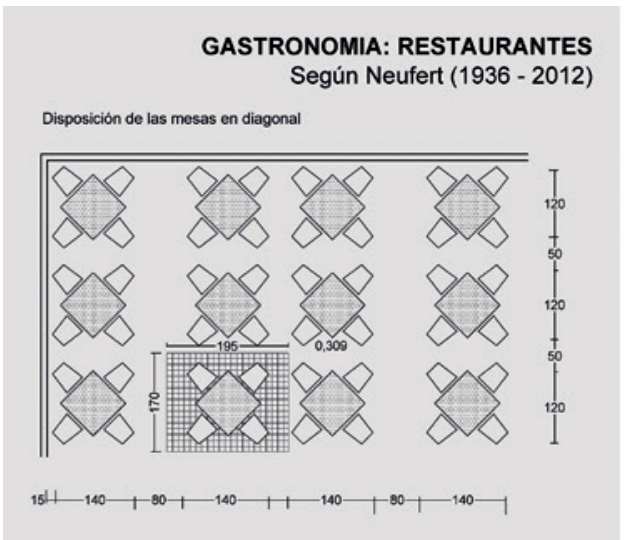

GASTRONOMIA: RESTAURANTES Variación COVID 2019

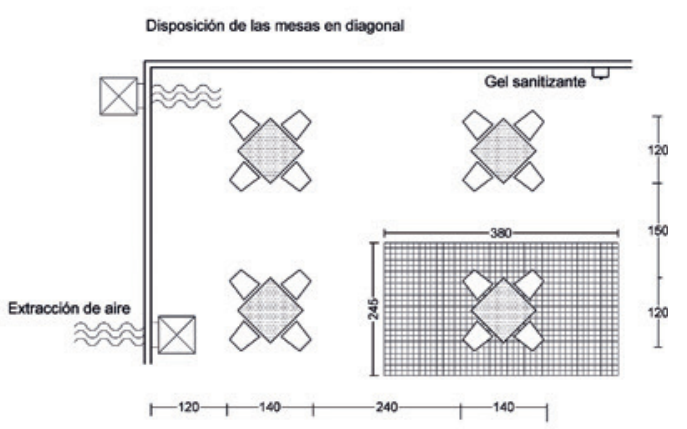


versión actualizada, en donde el contacto entre cuerpos se elimina del gráfico y se utilizan barreras transparentes para evitar la propagación de aerosoles entre feriantes y clientes, o para evitar que los productos queden expuestos a los transeúntes.

\section{DISCUSIÓN Y CONCLUSIONES}

Las ilustraciones anteriores, al comparar lo prescrito en el Neufert con su adaptación gráfica a lo prescrito en distintas normas y recomendaciones para la contingencia actual, indican una suspensión al menos temporal de la vigencia del manual arquitectónico. Las relaciones entre ocupación y espacio, las relaciones entre cuerpos próximos y capacidad del recinto dejan de estar vigentes como efecto de una especie de estado de guerra desatado por el COVID 19, y que ha dejado al Baventwurfslehre obsoleto en apenas unos meses, después de más de ochenta años de vigencia y metódica actualización. Pero en otro sentido, se identifica el fenómeno de una ciudad convertida en una suerte de nuevo Neufert en escala natural. Como es evidente, y como ha sido reconocido en distintas investigaciones, el uso del espacio ha cambiado, y la arquitectura prevé un conjunto de transformaciones desde la emergencia, que aunque pueden considerarse frágiles o efímeras, modifican la percepción y el uso del espacio (Ferrín-Salvador, 2020) En los espacios públicos y colectivos ha proliferado la aplicación de distintos esquemas y elementos gráficos que indican cuántos cuerpos pueden ocupar el espacio y cómo pueden hacerlo.

Líneas, círculos, flechas, textos y figuras humanas aparecen aplicados gráficamente sobre pavimentos y mobiliario urbano en todo espacio colectivo. Una suerte de representación arquitectónica esquemática, tan instrumental como la definida por Neufert, se apodera del espacio público (FIGURA 10). Esta operación de convertir la propia superficie de la ciudad y los edificios en superficie de representación de un esquema en escala natural está dando cuenta de un cambio sustancial en los modos de uso del espacio, sobre el que se desconoce
9. Izquierda: Recomendaciones de ocupación para venta ambulante de alimentos no preparados. Basado en Neufert (2013). p. 273. Derecha: Interpretación gráfica de las recomendaciones y dimensiones según el contexto de la Pandemia COVID 19 (dibujos del autor).

10. Aplicaciones de parámetros de distanciamiento y ordenamiento de los cuerpos en espacios colectivos. Fuente: fotografías de José Ignacio Vielma en Santiago de Chile.

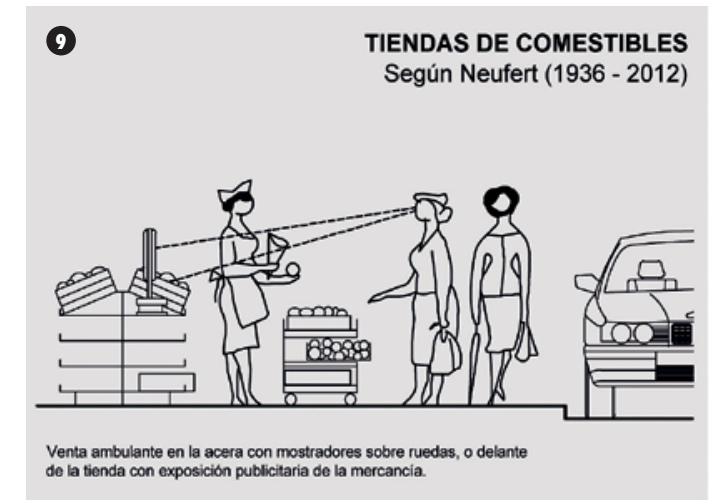

TIENDAS DE COMESTIBLES Variación COVID 2019
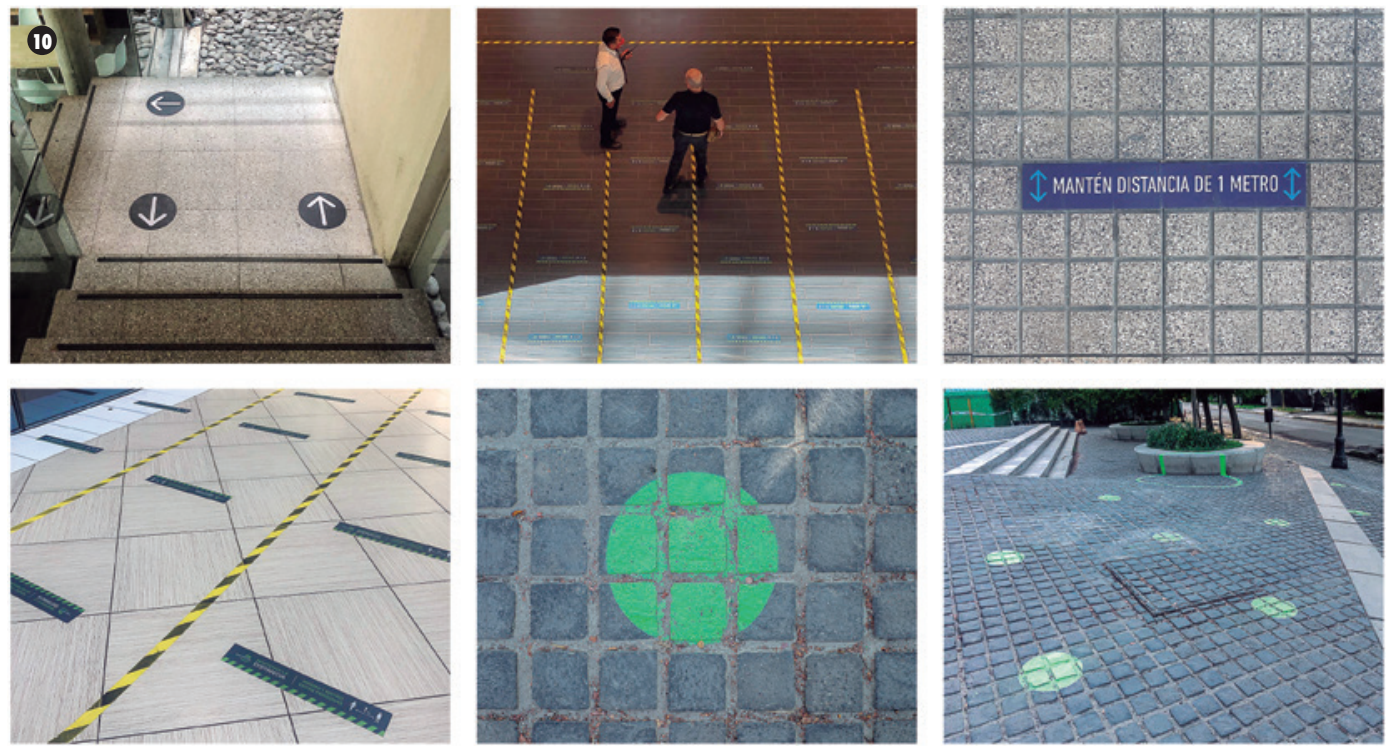

su duración y se teme su permanencia. Se vislumbra entonces, un modo completamente nuevo de habitar donde se impone la distancia, las barreras y los actos permanentes de desinfección y protección corporal.

Las infografías de El País referidas al principio fueron descritas por sus autores como el resultado de una "oportunidad visual". Eran muy elocuentes en explicar para todo público los procesos de contagio y su vinculación con cómo los cuerpos ocupan el espacio, pero representaban, sin saberlo, la continuidad de una instrumentalización en las relaciones entre cuerpo y arquitectura en favor de la higiene. El Baventwurfslehre de Neufert fundó esa tradición sustituyendo lo antropomórfico por lo antropométrico.

Las representaciones del Neufert aquí citadas o reintepretadas se basan también en ese oportunismo visual y se aprovechan de la pregnancia que en la disciplina tienen los dibujos de ese libro. Se puede valorar críticamente su realización y confrontación desde la evidencia, por una parte de lo antinatural de los nuevos modos de ocupar el espacio y de moverse que la pandemia ha instalado (Leiva 
et al., 2020), pero, por otra, de la insistente insuficiencia de la figura del cuerpo ocupante y estático para representar las complejidades del sujeto habitante.

Ahora bien, los gráficos presentados hasta ahora no deben tomarse en ningún caso como recomendaciones para proyectar en el contexto de pandemia. Su intención es llamar la atención, usando la analogía y simulando la objetividad que recrean, sobre lo inconveniente de prescribir a largo plazo, de asumir, en arquitectura, la inflexibilidad de las estructuras de conocimiento. Sirven para dar cuenta de cómo el escenario de la pandemia ha debilitado algunos territorios disciplinares que se daban por sentados, y que hasta por eso se consideraban irrelevantes, sin interés.

Las representaciones propuestas intentan llamar la atención sobre el espacio vacío que antes ocupaban las personas y sus interacciones, y de ningún modo intentar normar cómo llenar ese espacio de manera óptima o segura en el contexto de pandemia. Al confrontarlas sobre los discursos históricos o actuales de la relación de los cuerpos en el espacio, se hace evidente el descalce epistemológico entre dos modos de ver. Mientras Leonardo comprendía al cuerpo como referencia, o Solà-Morales e Imrie llamaban a su recuperación como depositario de fenómenos y experiencias, la antropometría ejemplificada en Neufert no solamente comprendía el cuerpo como una simple unidad de medida y cabida. También implicaba medir todo a partir de ese cuerpo originario e idealizado, y de conquistar el espacio necesario para su desarrollo, tal como fue pensado y ejecutado en el régimen nacionalsocialista al que el arquitecto alemán aportó parte de sus innovaciones. Esto debe servir de alerta a la instrumentalización irracional de las medidas, distancias y capacidades como factores únicos a considerar.

El resultado final de muchas de las políticas sanitarias que vinculan al cuerpo, su ocupación del espacio, y la enfermedad, ha sido lo que aquí se denomina "cuerpos ausentes", es decir, la baja utilización de los espacios públicos

11. Arriba: Recomendaciones de ocupación para distintos usos en espacios peatonales. Basado en Neufert (2013). p. 389. Derecha: Interpretación gráfica de las recomendaciones y dimensiones según el contexto de la Pandemia COVID 19. Fuente: dibujos del autor.

(1)

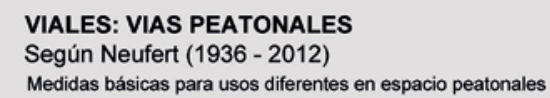

Medidas básicas para usos diferentes en espacio peatonales

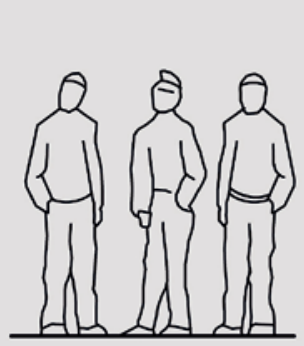

(19)

$1,5-2 \mathrm{~m}$ espacio necesario

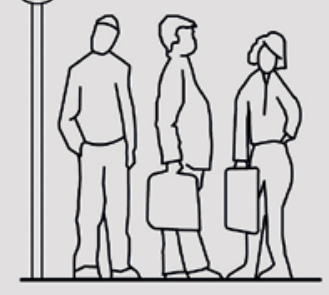

1,5 - 2 m espacio necesario

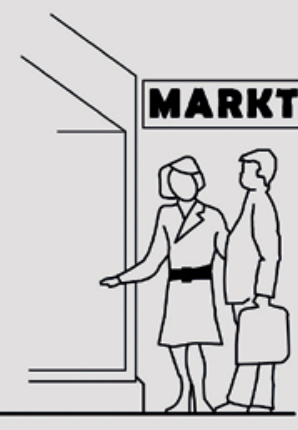

1,5 - 2 m espacio necesario

\section{VIALES: VIAS PEATONALES}

Variación COVID 2019

Medidas básicas para usos diferentes en espacio peatonales

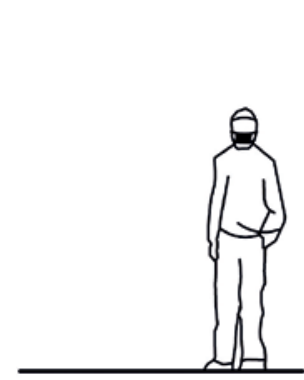

1,5 - 2 m espacio necesario
(4)

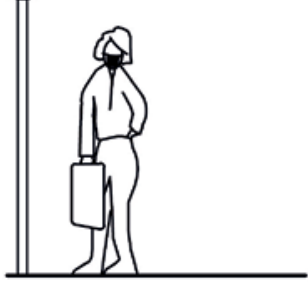

$1,5-2$ m espacio necesario

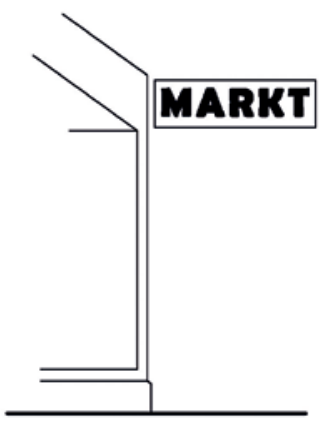

$0 \mathrm{~m}$ espacio necesario y colectivos, frecuente durante los períodos más radicales de cuarentena. La ciudad y los recintos vaciados y la ocupación racionalizada al máximo de espacios enormes anunciaron hace algunos meses un quiebre de relaciones racional y culturalmente establecidas y validadas por décadas. Aunque necesario en términos sanitarios, este abandono de la calle ha disminuido la sinergia y la copresencia, factores fundantes de lo urbano y el paisaje callejero (Soja, 2000), y ha obligado a establecer rituales privados que apenas hace poco nos podían resultar absurdos. Más grave aún, este impedimento para la copresencia urbana ha limitado el ejercicio económico y el acceso al trabajo, amenazando a las personas más vulnerables, los de la economía de calle o quienes realizan trabajos imposibles de realizar por telepresencia (Chirisa et al., 2020; Ziccardi, 2020). La FIGURA 11 expresa ese proceso, y puede servir para apoyar el cierre de este desarrollo. La ilustración original de Neufert definía las dimensiones necesarias de las vialidades peatonales según el número de personas o la actividad. Estar de pie o caminar, esperar el transporte, mirar vitrinas o consumir Posibilidad de relación con el otro, actividades constitutivas de una urbanidad elemental. En el contexto de la pandemia, una mayor distancia se hizo el fin último de la ocupación espacial, y la cercanía, que optimizaba el uso del espacio y propiciaba la interacción, se representa constantemente como inconveniente. Al inicio o en los momentos más radicales de la cuarentena, los pocos caminantes saltaban a la calzada o se alejaban rápidamente al ver que un cuerpo se aproximaba en sentido contrario. Los rebrotes en todo el mundo o las cifras consistentemente altas de entornos desregularizados como los Estados Unidos o 
Brasil, confirman la conveniencia sanitaria de una ciudad vacía, de la ausencia de los cuerpos, con una enorme cantidad de espacio usado por pocos. Allí se ha suspendido mucha de la antropometría conocida y nuevas relaciones de medida en relación con los cuerpos se han establecidos por medio de señaléticas que además recuerdan con insistencia el peligro de estar juntos.

\section{REFERENCIAS BIBLIOGRÁFICAS}

Chirisa, I., Mutambisi, T., Chivenge, M., Mabaso, E., Matamanda, A. R., y Ncube, R. (2020). The urban penalty of COVID- 19 lockdowns across the globe: manifestations and lessons for Anglophone sub-Saharan Africa. GeoJournal. https://doi. org/10.1007/s10708-020-10281-6

Ferrín-Salvador, M. (2020). Transformación de la arquitectura desde el COVID-19. Finibus, 3(6), 26-45. https://publicacionescd.uleam.edu.ec/ index.php/finibus/article/view/148/289

Gaytán Alcalá, F. (2020). Conjurar el miedo: El concepto Hogar - Mundo derivado de la pandemia COVID- 19. Relais, 3(1), 22-26.

Gesto, J. (2020). Repensando un nuevo modelo ciudad post-COVID-19. Designia, 8(2), 9-25. https://doi.org/https://doi. org/10.24267/22564004.604

Harvard Healthy Building Program, \& Health, H. T. H. C. S. of P. (2020). Guía en 5 pasos para medir la tasa de renovación de aire en aulas. Harvard T.H. Chan School of Public Health. https:// schools.forhealth.org/wp-content/uploads/ sites/19/2020/10/Harvard-Healthy-Buildingsprogram-How-to-assess-classroom-ventilation10-30-2020-ES.pdf
Imrie, R. (2003). Architects' conceptions of the human body. Environment and Planning D: Society and Space, 21 (1), 47-65. https://doi.org/10.1068/ d271t

Leiva, G. de C., Sathler, D., y Filho, R. D. O. (2020). Urban structure and population mobility: Implications for social distance and dissemination of COVID-19. Revista Brasileira de Estudos de Populacao, 37, 1-22. https://doi.org/10.20947/ s0102-3098a01 18

Ministerio de Educación-Chile (n.d.). Orientaciones COVID-19 para establecimeintos educacionales. Recuperado de https://www.mineduc.cl/ orientaciones-mineduc-COVID-19/

Ministerio de Salud-Chile (n.d.). Información técnica - Ministerio de Salud - Gobierno de Chile. Recuperado de https://www.minsal.cl/nuevocoronavirus-2019-ncov/informe-tecnico/

Ministerio de Salud-Chile (2020). Resolución Núm. 722 exenta. En Ministerio de Salud (Ed.), 722 (Vol. 722). Ministerio de Salud (Chile).

Neufert, E. (2013). Arte de proyectar en arquitectura (16a). Gustavo Gili.

Ortiz de Lejarazu, R. (2018). La pandemia de gripe española vista desde el siglo XXI. Anales de La Real Academia de Medicina y Cirugía de Valladolid, 55, 367-384.

Palero, J. S., \& Avila, M. (2020). COVID-19. La vigencia del higienismo decimonónico en tiempos de cuarentena. Cuaderno Urbano, 29(29). https://doi.org/10.30972/crn.29294620

Pernas Galí, F. (2020). La "arquitectura" de la COVID-19. Palimpsesto, 21, 6-7. https://upcommons.upc.edu/bitstream/ handle/2117/192512/9494-9611-1-PB. $\mathrm{pdf}$ ? sequence $=1$ \&isAllowed $=\mathrm{y}$

Shields, R., Schillmeier, M., Lloyd, J., \& Van Loon, J. (2020). 6 Feet Apart: Spaces and Cultures of Quarantine. Space and Culture 23 (3), pp. 216-220. https://doi. org/10.1177/1206331220938622
Soja, E. (2000). Postmetropolis: critical studies of cities and region. Blackwell.

Solà-Morales, I. (1996). Cuerpos ausentes. En Fudación Caja de Arquitectos (Ed.), Los artículos de Any-Ignasi de Solà-Morales, pp. 83-101. Fudación Caja de Arquitectos.

Vossoughian, N. (2015). From A4 paper to the Octametric brick: Ernst Neufert and the geopolitics of standardisation in Nazi Germany. Journal of Architecture, 20 (4), pp. 675-698. https://doi.org/10.1080/13602365.2015.107 2232

Vossoughian, N. (2016). Hausbaumaschine. Volume, $49,22-26$.

Vossoughian, N. (2020). Alvar Aalto, Ernst Neufert, and architectural standardization in Germany and Finland, 1933-45. Journal of the Society of Architectural Historians, 79(2), 202-212. https:// doi.org/10.1525/isah.2020.79.2.202

Zafra, M., \& Salas, J. (2020a). Radiografía de tres brotes: así se contagiaron y así podemos evitarlo. El País (España). https://elpais.com/ ciencia/2020-06-06/radiografia-de-tres-brotesasi-se-contagiaron-y-asi-podemos-evitarlo. $\mathrm{htm}$ ? $\mathrm{rel}=\mathrm{mas}$

Zafra, M., \& Salas, J. (2020b). Un salón, un bar y una clase: así contagia el coronavirus en el aire. El País (España). https://elpais.com/ ciencia/2020-10-24/un-salon-un-bar-y-unaclase-asi-contagia-el-coronavirus-en-el-aire.html

Ziccardi, A. (2020). Las grandes regiones urbanas y el distanciamiento social impuesto por el COVID-19. Astrolabio, 25, 46-64.https:// revistas.unc.edu.ar/index.php/astrolabio/article/ view $/ 29382 / 30827$

Zöllner, F. (2014). Anthropomorphism: From Vitruvius to Neufert, from Human Measurement to the Module of Fascism. Images of the Body in Architecture. Anthropology and Built Space, (July), pp. 47 - 75 